\title{
Aberrant expression of cytokine interleukin 9 along with interleukin 4 and interferon $\gamma$ in connective tissue disease-associated interstitial lung disease: association with severity of pulmonary fibrosis
}

Shan Jiang ${ }^{1}$, Zhi Wang ${ }^{1}$, Han Ouyang ${ }^{1}$, Zhichun Liư ${ }^{2}$ Lingyun Li ${ }^{3}$, Yongbing Shi ${ }^{1}$

\author{
${ }^{1}$ Department of Nephrology, The Second Affiliated Hospital of Soochow University, \\ Soochow, China \\ 2Department of Rheumatology, The Second Affiliated Hospital of Soochow University, \\ Soochow, China \\ ${ }^{3}$ Department of Pharmacology, Medical College of Soochow University, Soochow, \\ China
}

Submitted: 19 October 2013

Accepted: 7 February 2014

Arch Med Sci 2016; 12, 1: 101-106

DOI: $10.5114 /$ aoms.2015.47877

Copyright @ 2016 Termedia \& Banach

\section{Abstract}

Introduction: Connective tissues diseases (CTDS) are a heterogeneous group of disorders that share certain clinical characteristics and disturbed immunoregulation. Interstitial lung diseases (ILDs), also known as diffuse parenchymal lung diseases, are among the most serious pulmonary complications associated with CTDs. Interleukin 9 (IL-9), IL-4 and interferon $\gamma($ IFN- $\gamma)-c y-$ tokines with important roles in autoimmune disease - were studied in CTD patients and CTD-ILD patients.

Material and methods: Sixty-one hospitalized untreated CTD patients were recruited, and 20 healthy volunteers were enrolled as controls. The 61 CTD patients were divided into a simple CTD group and a CTD-ILD group, and the plasma protein IL-9, IL-4 and IFN- $\gamma$ levels were measured by enzyme-linked immunosorbent assay (ELISA).

Results: The results indicate that the serum IL-9 levels were significantly higher in CTD-ILD and simple CTD patients than they were in healthy controls (each $p<0.05$ ) and that the levels were elevated in CTD-ILD patients compared with simple CTD patients $(p<0.05)$. The IL-4 levels were higher in CTD-ILD patients than they were in the simple CTD patients $(p<0.05)$ and healthy controls $(p<0.01)$. In addition, the serum IL-9 levels were negatively correlated with the level of IFN- $\gamma\left(r^{2}=0.34, p=0.01\right)$, the estimated percentage of predicted forced vital capacity (FVC\%) $\left(r^{2}=0.36, p=0.00\right)$ and the estimated percentage of predicted diffusing capacity (DLCO\%) $\left(r^{2}=0.27\right.$, $p=0.04)$ and were positively correlated with the IL-4 level $\left(r^{2}=0.31, p=0.01\right)$. Conclusions: Interleukin-9 may play an important role in the pathogenesis of CTD and may contribute to the progression of interstitial lung injury in CTD patients.

Key words: interleukin 9, interleukin 4, interferon $\gamma$, connective tissues disease, interstitial lung disease.

\section{Introduction}

Connective tissues diseases (CTDs) include many types of autoimmune diseases. Interstitial lung diseases (ILDs), which are among the most seri-

\author{
Corresponding author: \\ Dr. Han Ouyang \\ Department of Nephrology \\ The Second Affiliated \\ Hospital \\ of Soochow University \\ 1055 San-Xiang Road \\ Suzhou 215004 \\ Jiangsu Province, China \\ Phone: +8667784159 \\ E-mail: ouyanghan198784@ \\ aliyun.com
}


ous pulmonary complications associated with CTDs, result in significant morbidity and mortality, a fact that should be well known to physicians. Connective tissues diseases and comorbid ILD are referred to as connective tissue disease-interstitial lung disease (CTD-ILD). Interstitial lung disease cases account for $15 \%$ of all respiratory system diseases [1]. Meanwhile, $15 \%$ of ILD cases are caused by CTD [2]. Although the pathogenesis of CTD and CTD-ILD remains unclear, many studies have suggested that cytokines or growth factors play an important role in the tissue injury, inflammation and fibrosis observed in CTD-ILD and that these factors may contribute to the immune dysfunction and mediation of the tissue inflammation and organ damage in the pathogenesis of CTD and CTD-ILD [3-5].

Interleukin 9 (IL-9) is a T cell-derived cytokine that was initially designated as a Th2 cytokine [6]. Interleukin 9 targets cells of the lymphoid, myeloid, and mast cell lineages and likely contributes to the development of autoimmune diseases [7-9]. A study by Evans et al. showed that the overexpression of IL-9 contributes to lung function damage [10]. However, whether the abnormal expression and secretion of IL-9 are present in CTD-ILD patients remains unknown. Therefore, in the present study, the expression of IL-9 levels in CTD-ILD patients and the association of IL-9 with disease activity were investigated to better understand the relationship and immunopathological role of IL-9 in CTD-ILD.

\section{Study cohort}

Peripheral blood samples were obtained from 61 patients with CTD in the Second Affiliated Hospital of Soochow University, including patients with systemic sclerosis (SSc), rheumatoid

Table I. Baseline patient characteristics

\begin{tabular}{|lccc|}
\hline Characteristics & $\begin{array}{c}\text { CTD } \\
\text { group }\end{array}$ & $\begin{array}{c}\text { CTD-ILD } \\
\text { group }\end{array}$ & HC \\
\hline Case number & 32 & 29 & 20 \\
\hline Age, mean \pm SD [years] & $36 \pm 12$ & $34 \pm 11$ & $31 \pm 7$ \\
\hline Male : female & $6: 26$ & $5: 24$ & $3: 17$ \\
\hline SSc, $n$ & 10 & 9 & 0 \\
\hline RA, $n$ & 8 & 7 & 0 \\
\hline DM, $n$ & 7 & 7 & 0 \\
\hline SLE, $n$ & 7 & 6 & 0 \\
\hline Decreased FVC\%, $n(\%)$ & $7(21.9)$ & $\begin{array}{c}21 \\
(72.4)\end{array}$ & 0 \\
\hline Decreased DL $\%, n(\%)$ & $8(25.0)$ & $\begin{array}{c}19 \\
(65.5)\end{array}$ & 0 \\
\hline
\end{tabular}

SSC - systemic sclerosis, RA - rheumatoid arthritis, DM - dermatomyositis, SLE - systemic lupus erythematosus, HC - healthy controls, FVC - forced vital capacity, $D L_{c o}$ - diffusing capacity. arthritis (RA), dermatomyositis (DM) and systemic lupus erythematosus (SLE). Determination of ILD was according to the chest high-resolution computed tomography (HRCT) performance, and the patients were divided into a CTD-ILD group and a simple CTD group according to presence or absence of ILD. The simple CTD group included 10 SSc patients, 8 RA patients, 7 DM patients, and 7 SLE patients, and the CTD-ILD group included 9 SSc patients, 7 RA patients, 7 DM patients, and 6 SLE patients. Patients did not have any acute infection and did not receive any immuno-modulating medication at the time of analysis. Twenty age- and sex-matched healthy Chinese individuals served as healthy controls. The clinical characteristics and patient demographics are shown in Table I. The study, informed consent forms and processes were approved by the involved institutions.

\section{Diagnostic criteria}

Patients with SSc fulfilled the criteria for SSc proposed by the American College of Rheumatology (ACR) [11]. The diagnosis of SLE was established by the presence of four or more American College of Rheumatology (ACR) diagnostic criteria [12], while patients with DM fulfilled the criteria of Bohan and Peter [13] and patients with RA fulfilled the proposed RA criteria [14]. The diagnosis of ILD was made using HRCT scanning, the results of which were interpreted by two expert thoracic radiologists. Lung involvement was observed using HRCT. Imaging findings included: reticulation, groundglass opacity, airspace consolidation, thickening of honeycombing, nodules, emphysema, bronchovascular bundle, bullae, traction bronchiectasis, crazy-paving appearance, tree-in-bud appearance, bronchiectasis or bronchiolectasis, mosaic perfusion, architectural distortion, and air trapping [15].

\section{Material and methods}

\section{Measurement of serum IL-9, IL-4 and IFN- $\gamma$} levels

Venous blood samples were drawn into pyrogen-free blood collection tubes, immediately immersed in melting ice, and allowed to clot for $1 \mathrm{~h}$ before centrifugation. All serum samples were stored at $-80^{\circ} \mathrm{C}$ before use. All procedures were standardized. The serum IL-9, IL-4 and interferon gamma (IFN- $\gamma$ ) levels were measured using specific ELISA kits (R\&D Systems, Minneapolis, MN, USA). Each sample was tested in duplicate. The results were expressed as picograms per milliliter, with an assay detection limit of $0.5 \mathrm{pg} / \mathrm{ml}$.

\section{Pulmonary function test}

A pulmonary function test (PFT), including the estimated percentage of predicted forced vital 
capacity (FVC\%) and the estimated percentage of predicted diffusing capacity $\left(\mathrm{DL}_{\mathrm{co}} \%\right)$, was evaluated to determine the severity of pulmonary fibrosis. A pulmonary function test measurements were obtained using a lung function instrument (VMAX21-1, Sensormedics, USA). Forced vital capacity and $\mathrm{DL}_{\mathrm{CO}}$ measurements were considered to be abnormal at $<80 \%$ and $<75 \%$ of the predicted normal values, respectively. Patients with CTD who were smokers or had other respiratory disorders that could have affected $\mathrm{DL}_{\mathrm{CO}} \%$ or $\mathrm{FVC} \%$ were excluded from our study.

\section{Statistical analysis}

The results are expressed as the mean \pm standard deviation (SD). The statistical analysis of the data was performed using the GraphPad Prism (version 4.0) statistical program. The nonparametric Mann-Whitney $U$-test was used to compare data between two groups. An analysis of covariance was used to compare data between the three groups. For the correlation analysis with laboratory parameters, Pearson's correlation coefficient was used. Two-sided $p$-values $<0.05$ were considered to indicate a statistically significant difference.

\section{Results}

\section{Imaging findings of chest HRCT of CTD-ILD patients}

The HRCT findings were observed in 29 CTD-ILD patients. Reticulation and ground-glass opacity were the most frequent findings in CTD-ILD pa-

Table II. Imaging findings of chest HRCT in CTD-ILD patients

\begin{tabular}{|c|c|}
\hline Imaging findings & $\begin{array}{l}\mathrm{HRCT} \\
n(\%)\end{array}$ \\
\hline Reticulation & $17(58.6)$ \\
\hline Ground-glass opacity & $15(51.7)$ \\
\hline Airspace consolidation & $4(13.8)$ \\
\hline Thickening of bronchovascular bundle & $1(3.4)$ \\
\hline Honeycombing & $7(24.1)$ \\
\hline Nodules & $5(17.2)$ \\
\hline Crazy-paving appearance & $1(3.4)$ \\
\hline Bronchiectasis or bronchiolectasis & $2(6.9)$ \\
\hline Bullae & $1(3.4)$ \\
\hline Tree-in-bud appearance & $2(6.9)$ \\
\hline Mosaic perfusion & $1(3.4)$ \\
\hline Traction bronchiectasis & $10(34.5)$ \\
\hline Architectural distortion & $6(20.7)$ \\
\hline Air trapping & $3(10.3)$ \\
\hline Emphysema & $2(6.9)$ \\
\hline
\end{tabular}

tients. Honeycombing, traction bronchiectasis, and architectural distortion were also frequently observed. Nodules were observed in 5 patients, airspace consolidation in 4 patients and air trapping in 3 patients. The frequency of the HRCT findings is summarized in Table II.

\section{Serum IL-9 levels elevated in the simple CTD group and the CTD-ILD group}

To define abnormalities in serum IL-9 expression in the peripheral blood of CTD and CTD-ILD patients, we tested the serum IL-9 levels from the peripheral blood of all 61 CTD patients. We found that the serum IL-9 levels were significantly higher in CTD-ILD and simple CTD patients than they were in healthy controls $(78.9 \pm 16.9 \mathrm{pg} / \mathrm{ml}$ vs. 13.7 $\pm 5.5 \mathrm{pg} / \mathrm{ml}, p<0.05 ; 40.2 \pm 8.1 \mathrm{pg} / \mathrm{ml}$ vs. $13.7 \pm 5.5$ $\mathrm{pg} / \mathrm{ml}, p<0.05)$ and that the levels were elevated in CTD-ILD patients compared with simple CTD patients $(78.9 \pm 16.9 \mathrm{pg} / \mathrm{ml}$ vs. $40.2 \pm 8.1 \mathrm{pg} / \mathrm{ml}$, $p<0.05)$ (Figure 1).

\section{Serum IL-4 and IFN- $\gamma$ levels in the simple CTD group, CTD-ILD group and healthy controls}

Next, to determine whether the expression of IL-4 and IFN- $\gamma$ was altered in the CTD-ILD patients, we tested the serum IL-4 and IFN- $\gamma$ levels in simple CTD patients, CTD-ILD patients and healthy controls. The results showed that the IL-4 levels were higher in CTD-ILD patients than they were in the simple CTD patients and healthy controls (74.6 $\pm 14.7 \mathrm{pg} / \mathrm{ml}$ vs. $43.6 \pm 7.9 \mathrm{pg} / \mathrm{ml}, p<0.05 ; 74.6$ $\pm 14.7 \mathrm{pg} / \mathrm{ml}$ vs. $13.9 \pm 4.9 \mathrm{pg} / \mathrm{ml}, p<0.01$ ) (Figure 2). The IFN- $\gamma$ levels were lower in CTD-ILD patients

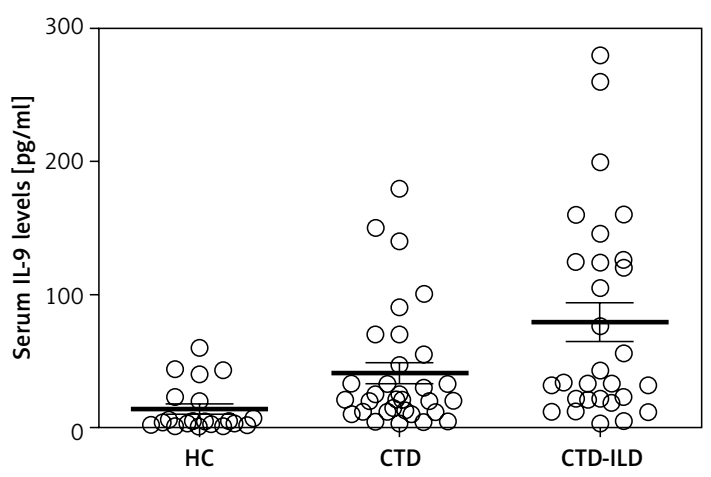

Figure 1. Serum IL-9 protein levels. The serum IL-9 levels in each group, including CTD-ILD patients (CTD-ILD group, $n=29$ ), simple CTD patients (simple CTD group, $n=32$ ) and healthy controls (HC, $n=20$ ), were detected by a specific ELISA. Bars indicate the mean values \pm SD in each group. The serum IL-9 levels were significantly higher in CTDILD and simple CTD patients compared to healthy controls ( $p<0.05$ for each) and were elevated in CTD-ILD patients compared to simple CTD patients $(p<0.05)$ 


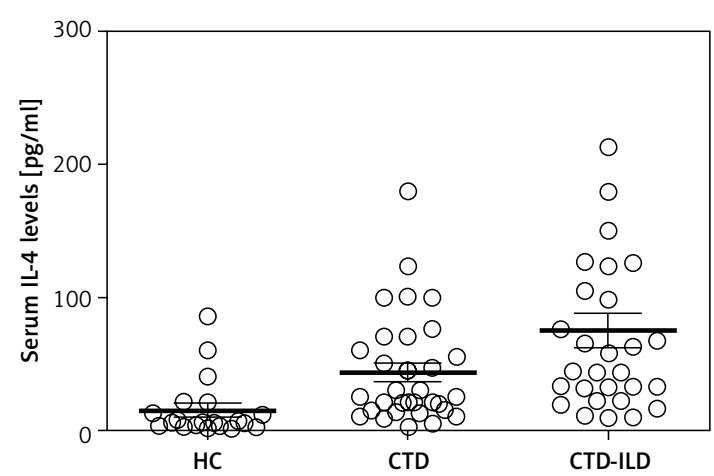

Figure 2. Serum IL-4 protein levels. The serum IL-4 levels were higher in CTD-ILD patients (CTD-ILD group, $n=29$ ) compared to simple CTD patients (simple CTD group, $n=32$ ) and healthy controls $(\mathrm{HC}, n=20)(p<0.05 ; p<0.01)$. The serum IL-4 levels were significantly increased in simple CTD patients compared to healthy controls $(p<0.05)$ Bars represent the mean values \pm SD

than they were in simple CTD patients $(18.6 \pm 4.2$ $\mathrm{pg} / \mathrm{ml}$ vs. $33.2 \pm 6.1 \mathrm{pg} / \mathrm{ml}, p<0.05)$, and the IFN- $\gamma$ levels were higher in simple CTD patients than they were in healthy controls $(33.2 \pm 6.1 \mathrm{pg} / \mathrm{ml}$ vs. $14.2 \pm 6.0 \mathrm{pg} / \mathrm{ml}, p<0.05)$, as shown in Figure 3 .

\section{CTD patients with pulmonary function testing}

The pulmonary function of 29 CTD-ILD patients and 32 simple CTD patients was tested using the lung function instrument. The results show that $72.4 \%$ of the CTD-ILD patients had decreased FVC\% and that $65.5 \%$ of the CTD-ILD patients had decreased $\mathrm{DL}_{\mathrm{CO}} \%$. Moreover, pulmonary function decline was evident in a handful of simple CTD patients, as shown in Table I.

\section{Increased IL-9 levels are associated with higher frequency and severity of pulmonary fibrosis}

To determine whether serum IL-9 levels were associated with the severity of pulmonary fibrosis in CTD-ILD patients, we correlated the serum IL-9 levels with some laboratory parameters. The results showed that the serum IL-9 levels were negatively correlated with $\mathrm{FVC} \%$ and $\mathrm{DL}_{\mathrm{CO}} \%$ in

Table III. Correlation of serum IL-9 levels with FVC\%, $\mathrm{DL}_{\mathrm{co}} \%$, serum IL-4 levels and serum IFN- $\gamma$ levels in patients with CTD

\begin{tabular}{lcccc|}
\hline Categories & IL-4 & IFN- $\gamma$ & FVC\% & DL $_{\mathrm{CO}} \%$ \\
\hline$r^{2}$ & 0.31 & 0.34 & 0.36 & 0.27 \\
\hline Values of $p$ & 0.01 & 0.01 & 0.00 & 0.04 \\
\hline
\end{tabular}

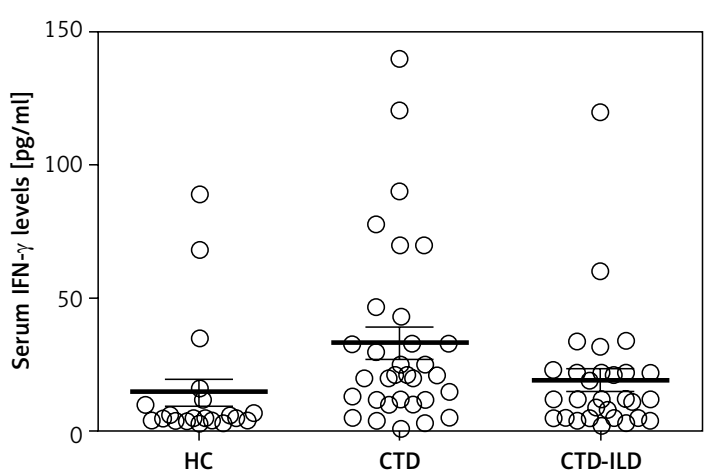

Figure 3. Serum IFN- $\gamma$ protein levels. This figure shows that the serum IFN- $\gamma$ protein levels were lower in CTD-ILD patients (CTD-ILD group, $n=29$ ) compared to simple CTD patients (simple CTD group, $n=32)(p<0.05)$ and that the serum IFN- $\gamma$ levels were higher in simple CTD patients compared to healthy controls (HC, $n=20)(p<0.05)$. There was no significant difference between the CTD-ILD group and healthy controls $(p>0.05)$

patients with CTD $\left(r^{2}=0.36, p<0.01 ; r^{2}=0.27\right.$, $p<0.01$; Table III). Further, we investigated the correlation between serum IL-9 levels and serum IFN- $\gamma$ and serum IL-4 levels, which are markers of pulmonary fibrosis, in patients with CTD-ILD. We found that the serum IL-9 levels were inversely correlated with serum IFN- $\gamma$ levels $\left(r^{2}=0.34\right.$, $p<0.01)$ and positively correlated with IL-4 levels $\left(r^{2}=0.31, p<0.05\right)$ (Table III).

\section{Discussion}

Connective tissues diseases encompass many types of diseases, including SLE, SSc, RA, primary Sjogren's syndrome (pSS), and DM. Interstitial lung diseases, which are also known as diffuse parenchymal lung diseases, are a heterogeneous group of pulmonary disorders with substantial morbidity and mortality. Connective tissues diseases often involve the lungs, and in DM patients, lung involvement is most commonly observed as visceral lesions, which can seriously impact a patient's prognosis and may appear at any time in the course of DM-ILD [16]. Rheumatoid arthritis is the most common form of CTD, and more than half of the patients with RA present with ILD [17]. Patients with SSc have an even higher incidence of ILD, which is a leading cause of death in SSc patients; ILD is part of the secondary diagnostic criteria of SSc [18]. Castelino et al. reported that the incidence of ILD in SSc, DM, RA, pSS, mixed connective tissue disease (MCTD) and SLE patients is $65 \%, 40 \%, 35 \%, 25 \%, 65 \%$ and $10 \%$, respectively [19]. Several studies have indicated that ILD is an important complication of CTD.

At present, the pathogenesis of CTD-ILD is not well understood. However, many studies have shown that Th1, Th2, Th17 cells and abnormal- 
ities of their associated cytokines may be related to the occurrence of CTD-ILD [20-22]. Studies have shown that $\mathrm{CD}^{+}{ }^{+} \mathrm{T}$-cell subsets, including Th1, Th2, Th17 and Th9, can produce IL-9 under specific conditions and that the overexpression of IL-9 is involved in the development of pulmonary fibrosis and associated with the severity of lung fibrosis in mice $[23,24]$. This evidence indicated that elevation of IL-9 levels may be implicated in the pathogenesis of CTD patients with pulmonary fibrosis. Pulmonary fibrosis is a parenchymal lung disease characterized by progressive interstitial fibrosis [25]. In the present study, plasma concentrations of three Th-associated cytokines, IL-9, IL-4 and IFN- $\gamma$, were determined. We first found that the IL-9 levels were elevated not only in patients with simple CTD but also in those with CTD-ILD, demonstrating that IL-9 may play an important role in the pathogenesis of CTD and may contribute to the progression of interstitial lung injury in CTD patients.

Interleukin 4 can facilitate the formation of pulmonary fibrosis by promoting fibroblast proliferation, collagen synthesis and locomotion of the fibroblasts, while elevated IFN- $\gamma$ levels can protect against the development of pulmonary fibrosis by weakening fibroblast proliferation and collagen deposition. To elucidate whether the expression of IL-4 and IFN- $\gamma$ is abnormal in patients with SLE, serum IL-4 and IFN- $\gamma$ levels were analyzed in patients with simple CTD and CTD-ILD. Consistent with higher plasma IL-9 levels, our results have demonstrated that CTD-ILD patients also had increased plasma IL-4 levels compared with simple CTD patients, and that the IFN- $\gamma$ levels are significantly decreased in CTD-ILD patients compared with simple CTD patients. These findings are in accordance with the results reported by Huaux et al. [26] and Kunisawa et al. [3]. Meanwhile, we found a strong positive correlation between IL-9 levels and IL-4 levels and a negative correlation between the serum levels of IL-9 and serum levels of IFN- $\gamma$ in all CTD patients, suggesting that IL-9 production in CTD serves as a pro-inflammatory factor that functions in a similar fashion as IL-4. The fibrosis-promoting role of IL-9 in CTD patients may be related to B cells. Past evidence demonstrates that the overexpression of IL-9 enhances the recruitment of $B$ cells in the lung in murine pulmonary fibrosis [24]. However, some reports show that increased IL-9 can induce IL-17 production and regulatory $T$ cell activation, thereby inhibiting the development of pulmonary fibrosis in CTD [27]. Thus, the functions and mechanisms of IL-9 in CTD have yet to be clarified.

Restrictive ventilatory dysfunction is the most common pulmonary function injury in CTD patients, with FVC and $\mathrm{DL}_{\mathrm{co}}$ decline often appearing in the early stages of the disease. Because $\mathrm{FVC}$ and $\mathrm{DL}_{\mathrm{co}}$ testing can determine the severity of lung injury, we conducted pulmonary function testing in all CTD patients. Our results showed that a decrease in both FVC and $\mathrm{DL}_{\mathrm{co}}$ was present in $72.4 \%$ and $65.5 \%$ of CTD-ILD patients, respectively, demonstrating that the vast majority of CTD-ILD patients have lung function damage. In addition, a small number of patients with simple CTD also had abnormal lung function, which was thought to be related to pulmonary hypertension. This finding is indirect evidence that impaired lung function in patients with simple CTD appears earlier than do chest CT findings. The serum IL-9 levels were negatively correlated with the FVC and $D_{\text {co }}$ results, which indicates that the IL-9 levels are gauges of the degree of damage to pulmonary function in patients with CTD-ILD. The mechanism of injury may be that high expression of IL-9 increases the release of mucus from airway epithelial cells, which is related to airway hyperresponsiveness [28]. However, the precise mechanisms are undefined, and further studies are required.

In conclusion, this study demonstrates that serum IL-9 levels and IL-4 levels were elevated in patients with CTD, particularly those with CTD-ILD, and that IL-9 levels were associated with a higher prevalence of pulmonary involvement and worse pulmonary function. Thus, IL-9 may have a significant role in the development of pulmonary fibrosis in CTD and may contribute to the progression of interstitial lung injury in patients with CTD. Further studies are required to investigate the signaling transduction mechanisms and the precise effect of IL-9 in the pathogenesis of CTD and CTD-ILD. Targeting IL-9 and IL-4 in human CTD and CTDILD could be a promising approach in the future. Therefore, further investigations are warranted to confirm and build upon the findings of this study.

\section{Acknowledgments}

Shan Jiang and Zhi Wang contributed equally to this study.

This study was partly supported by the National Natural Science Foundation of China (grant no. 81302584, grant no. 81200507) and the Natural Science Foundation of Jiangsu Province (grant no. SBK201121707).

\section{Conflict of interest}

The authors declare no conflict of interest.

\section{References}

1. The diagnosis, assessment and treatment of diffuse parenchymal lung disease in adults. Introduction. Thorax 1999; 54 Suppl. 1: S1-14.

2. Strange C, Highland KB. Interstitial lung disease in the patient who has connective tissue disease. Clin Chest Med 2004; 25: 549-59. 
3. Kunisawa A, Kawanishi Y, Tago H, Nagate A, Kasuga I, Ohyashiki K. Direct demonstration of the productive capability of cytokines at the single cell level in lung sarcoidosis using multicolor cytometry. Respiration 2002; 69: 155-64.

4. Antoniou KM, Ferdoutsis E, Bouros D. Interferons and their application in the diseases of the lung. Chest 2003; 123: 209-16.

5. Dziankowska-Bartkowiak B, Gerlicz-Kowalczuk Z, Waszczykowska E. Angiogenin and SDF-1alpha serum concentration in patients with systemic sclerosis in relation to clinical status. Arch Med Sci 2011; 7: 92-6.

6. Knoops L, Renauld JC. IL-9 and its receptor: from signal transduction to tumorigenesis. Growth Factors 2004, 22: 207-15.

7. Soroosh P, Doherty TA. Th9 and allergic disease. Immunology 2009; 127: 450-8.

8. Nowak EC, Noelle RJ. Interleukin-9 as a T helper type 17 cytokine. Immunology 2010; 131: 169-73.

9. Ouyang H, Shi YB, Liu ZC, et al. Increased interleukinand CD4+IL-9+T cells in patients with systemic lupus erythematosus. Mol Med Rep 2013; 7: 1301-7.

10. Evans CM, Kim K, Tuvim MJ, Dickey BF. Mucus hypersecretion in asthma: causes and effects. Curt Opin Pulm Med 2009; 15: 4-11.

11. Subcommittee for Scleroderma Criteria of the American Rheumatism Association Diagnostic and Therapeutic Criteria Committee. Preliminary criteria for the classification of systemic sclerosis (scleroderma). Arthritis Rheum 1980; 23: 581-90.

12. Tan EM, Cohen AS, Fries JF, et al. The 1982 revised criteria for the classification of systemic lupus erythematosus. Arthritis Rheum 1982; 25: 1271-7.

13. Bohan A, Peter JB. Polymyositis and dermatomyositis (first of two parts). N Engl J Med 1975; 292: 344-8.

14. Arnett FC, Edworthy SM, Bloch DA, et al. The American Rheumatism Association 1987 revised criteria for the classification of rheumatoid arthritis. Arthritis Rheum 1988; 31: 315-24.

15. Tanaka N, Kim JS, Newell JD, et al. Rheumatoid arthritis-related lung diseases: CT findings. Radiology 2004 32: 81-91.

16. Arsura EL, Greenberg AS. Adverse impact of interstitia pulmonary fibrosis on prognosis in polymyositis and dermatomyoaitis. Semin Arthritis Rheum 1998; 18: 29-37.

17. Brown KK. Rheumatoid lung disease. Proc Am Thorac Soc 2007; 4: 443-8.

18. Tyndall AJ, Bannert B, Vonk $M$, et al. Causes and risk factors for death in systemic sclerosis: a study from the EULAR Selemderma Trials and Research (EUSTAR) database. Ann Rheum Dis 2010; 69: 1809-15.

19. Castelino FV, Varga J. Interstitial lung disease in connective tissue diseases: evolving concepts of pathogenesis and management. Arthritis Res Ther 2010; 12: 213.

20. Bilgic $H$, Ytterberg SR, Amin S, et al. Interleukin-6 and type I interferon-regulated genes and chemokines mark disease activity in dermatomyositis. Arthritis Rheum 2009; 60: 3436-46.

21. Gone T, Kawaguehi Y, Sugiura T, et al. Interleukin-18 is a key mediator in dermalamyositis: potential contribu tion to development of interstitial lung disease. Rheumatology (Oxford) 2010; 49: 1878-81.

22. Homer RJ, Zheng T, Chupp G, et al. Pulmonary type II cell hypertrophy and pulmonary lipoproteinosis are features of chronic IL-13 exposure. Am J Physiol Lung Cell Mol Physiol 2002; 283: L52-9.
23. van den Brûle S, Heymans J, Havaux X, et al. Profibrotic effect of IL-9 overexpression in a model of airway remodeling. Am J Respir Cell Mol Biol 2007; 37: 202-9.

24. Arras $M$, Huaux F, Vink $A$, et al. Interleukin-9 reduces lung fibrosis and type 2 immune polarization induced by silica particles in a murine model. Am J Respir Cell Mol Biol 2001; 24: 368-75.

25. Gao X, Wang G, Zhang W, Peng Q, Xue M, Jinhong H. Expression of pulmonary aquaporin 1 is dramatically upregulated in mice with pulmonary fibrosis induced by bleomycin. Arch Med Sci 2013; 9: 916-21.

26. Huaux F, Tian J, Liu M, Ullenbruch M, Phan SH. Dual roles of IL-4 in lung injury and fibrosis. J Immunol 2003; 13 . 124-5.

27. Koichi Y, Ayumi Y, Yoshihide A, Kadono T, Sato S. Serum interleukin 9 levels are increased in patients with systemic sclerosis: association with lower frequency and severity of pulmonary fibrosis. J Rheumatol 2011; 38 : 2193-7.

28. Steenwinckel V, Louahed J, Orabona C, et al. IL-13 mediates in vivo IL-9 activities on lung epithelial cells hut not on hematopoietic cells. J Immunol 2007; 178: 3244-51. 